\title{
ENGENHARIA E DESIGN DE INTERESSE SOCIAL: FORMULAÇÃO DE PROJETOS EM CARREIRAS DE ENGENHARIA NO ENSINO À DISTÂNCIA UTILIZANDO DESIGN THINKING
}

DOI: 10.37702/2175-957X.COBENGE.2021.3691

Ari Reis Frutuoso - ari.frutuoso@gmail.com

Universidade Virtual do Estado de São Paulo

Estrada São Francisco 1588

06765-000 - Taboão da Serra - SP

David Alejandro Lazo Vásquez - lazovasquez@usp.br

Universidade de São Paulo

Martim Francisco 159

01226-001 - Sao Paulo - SP

Resumo: A interdisciplinaridade é fundamental no processo de aprendizagem em carreiras de engenharia. A metodologia de Design Thinking permite desenvolver projetos focados nas necessidades dos usuários, possibilitando a interação entre alunos e a comunidade. Este artigo propõe uma metodologia para a formulação, desenvolvimento e análise de projetos no ensino de engenharia a distância utilizando Design Thinking, integrando engenharia, design e comunidade. Por meio de um estudo de caso na cidade de Taboão da Serra, a metodologia proposta foi utilizada para desenvolver um programa de capacitação para uma cooperativa de reciclagem, seguindo a norma NBR ISO 10015:2020. A principal contribuição deste trabalho é um modelo sistematizado para desenvolver projetos multidisciplinares em carreiras de engenharia, superando as limitações do ensino à distância e exercendo a vantagem de uma comunicação efetiva entre os alunos, o professor-tutor e os usuários.

Palavras-chave: Design Thinking, Empreendedorismo, Ensino a Distância, Ensino em engenharia, Inovação. 


\section{ENGENHARIA E DESIGN DE INTERESSE SOCIAL: FORMULAÇÃO DE PROJETOS EM CARREIRAS DE ENGENHARIA NO ENSINO A DISTÂNCIA UTILIZANDO DESIGN THINKING}

\section{INTRODUÇÃO}

Atualmente, o mercado exige profissionais de engenharia com habilidades de comunicação, capacidade de trabalhar em equipe (ZINDEL et al., 2012). No Brasil, nos últimos anos, o número de Instituições de Ensino Superior que oferecem cursos de engenharia aumentou consideravelmente, assim como também o número de vagas ofertadas (INEP, 2016). Conforme Zindel et al. (2012), a inovação curricular busca formar profissionais com uma abordagem baseada em projetos, cujas estratégias de aprendizagem ativa sejam associadas a experiências contextuais significativas, onde 0 aluno se envolve ativamente no processo de aprendizagem. Villarroel e Ossandón (1988) apontam o desafio enfrentado pelas escolas de engenharia nos países em desenvolvimento para acompanhar as necessidades locais de desenvolvimento.

Segundo Lima, Mesquita e Coelho (2017), a maioria dos atuais professores do ensino superior não teve estratégias ativas de aprendizagem durante sua formação. Assim, apoiar os professores na implementação de estratégias ativas de aprendizagem é uma necessidade. A interdisciplinaridade é fundamental no planejamento das atividades desenvolvidas na sala de aula. Na modalidade de ensino à distância (EaD), o professortutor acompanha, orienta, e avalia os alunos em suas atividades através de um ambiente virtual de aprendizagem (CARMO e FRANCO, 2019), sendo necessário desenvolver práticas pedagógicas através de recursos digitais. Segundo Giorgetti (1993), a evolução dos sistemas educacionais depende de suas interações com o meio socioeconômico. Portanto, a integração entre o design de interesse social e a engenharia é essencial no processo de formação de engenheiros no mundo contemporâneo (MONTEIRO e BARTHOLO, 2007). Nos últimos meses, devido ao avanço da pandemia da COVID 19, o uso de ferramentas on-line no ensino presencial e à distância ganharam força, evidenciando suas vantagens.

Este estudo propõe uma metodologia para a formulação, desenvolvimento e análise de projetos de interesse social por alunos de engenharia na modalidade EaD através de atividades que incentivam a integração de design e engenharia. A estrutura deste trabalho é a seguinte. Na seção 2, utilizando a metodologia Design Thinking, apresenta-se a metodologia proposta, incluindo as etapas necessárias para a definição do escopo de um projeto, o planejamento das atividades dos alunos e a organização do acompanhamento por parte do professor-tutor. A seção 3 mostra a aplicação da metodologia através de um estudo de caso desenvolvido na Universidade Virtual do Estado de São Paulo (UNIVESP) na cidade de Taboão da Serra. $\mathrm{Na}$ seção 4, apresentam-se as considerações finais, destacando as vantagens da adoção desta metodologia em cursos de engenharia em modalidade EaD e presencial.

\section{MÉTODOS}

A metodologia de Design Thinking (BROWN e WYATT, 2010), é amplamente utilizada na área do Design, no entanto, nos últimos anos tem sido empregada na formulação de projetos em diversas áreas do conhecimento facilitando 0 
empreendedorismo social. Esta proposta é baseada nos trabalhos de Monteiro e Bartholo (2007), Monteiro (2008), Brown e Wyatt (2010) e Stainbeck (2011), os quais são adaptados à modalidade de $\mathrm{EaD}$.

\subsection{Formulação de projetos de interesse social}

A escolha das atividades para o planejamento de projetos de interesse social depende de vários fatores, incluindo a formação dos alunos, o contexto de aplicação, o tempo de planejamento, o orçamento para o desenvolvimento do projeto no futuro, e outros. Um dos fatores essenciais no planejamento das atividades é a orientação continua dos alunos, dado que, muitos alunos possuem um tempo limitado para participar das atividades. A Tabela 1 mostra as atividades necessárias para a formulação de um projeto de interesse social, no estado de São Paulo, no contexto da EaD.

Tabela 1 - Atividades necessárias para formular projetos de interesse social.

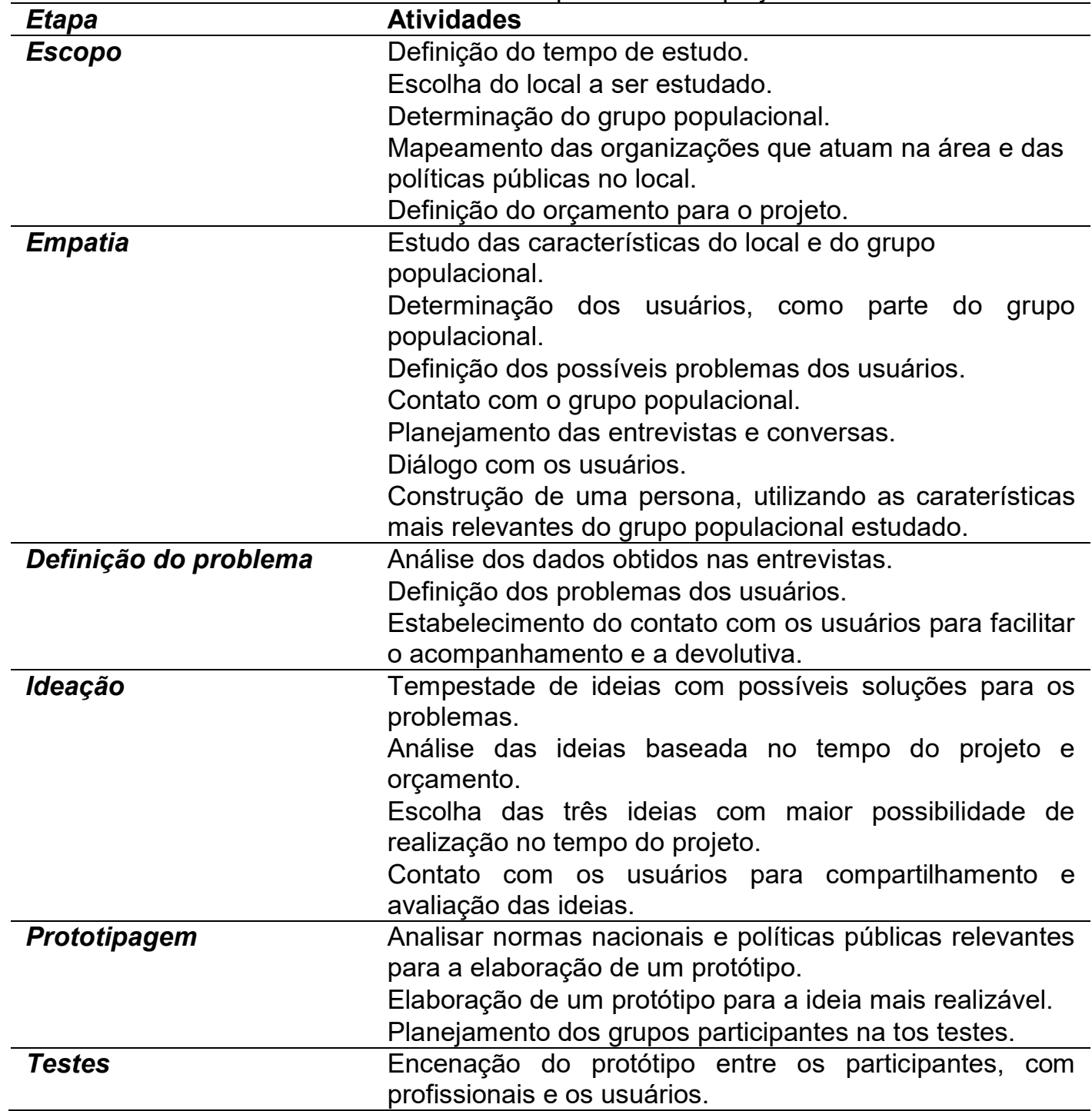

Fonte: Elaboração própria.

O acompanhamento do professor-tutor no desenvolvimento das atividades visa manter os alunos no escopo e providenciar referencias e ferramentas para a sistematização da informação obtida. É essencial manter o contato com o grupo populacional durante todas 
as etapas para facilitar a devolutiva e evitar que o projeto não satisfaça as reais necessidades dos usuários.

\subsection{Planejamento de atividades e acompanhamento dos alunos}

Com o fim de acompanhar o avanço dos alunos no cumprimento das atividades mostradas na Tabela 1, a cada duas semanas, conteúdos relacionados às etapas do Design Thinking (ver Tabela 2) são lançados através de uma plataforma on-line. $O$ projeto é realizado em 16 semanas.

Tabela 2 - Atividades para o acompanhamento dos alunos.

\begin{tabular}{|c|c|c|}
\hline Quinzena & Módulos & Atividades \\
\hline 1 & $\begin{array}{l}\text { Os reflexos de uma sociedade } \\
\text { competitiva e globalizada na ocupação } \\
\text { dos espaços urbanos públicos e privados }\end{array}$ & $\begin{array}{l}\text { Aproximação ao tema, escolha do } \\
\text { local e observação in loco }\end{array}$ \\
\hline 2 & $\begin{array}{l}\text { Coleta de Informações: visitando o } \\
\text { espaço e ouvindo seus integrantes e } \\
\text { especialista }\end{array}$ & Definição e estudo sobre o problema \\
\hline 3 & Definindo um problema a estudar & $\begin{array}{l}\text { Visita a campo e definição da } \\
\text { solução }\end{array}$ \\
\hline 4 & $\begin{array}{l}\text { Apresentando os primeiros resultados do } \\
\text { seu Projeto Integrador }\end{array}$ & $\begin{array}{l}\text { Elaboração da solução e visita a } \\
\text { campo }\end{array}$ \\
\hline 5 & $\begin{array}{l}\text { Formulando a proposta ou projeto de } \\
\text { análise inicial }\end{array}$ & $\begin{array}{l}\text { Entrega da primeira versão da } \\
\text { solução }\end{array}$ \\
\hline 6 & $\begin{array}{l}\text { Analisando dados e formulando a } \\
\text { proposta }\end{array}$ & Devolutiva do mediador \\
\hline 7 & Redigindo o relatório final relatório final & Melhoria da solução \\
\hline 8 & Entregando o relatório final relatório final & Entrega da solução final \\
\hline
\end{tabular}

Fonte: Elaboração própria.

O processo envolve o surgimento de inconvenientes próprios do EaD e da interação com o grupo populacional sob estudo. Os tempos planejados na etapa inicial do projeto podem ser afetados pela disponibilidade dos usuários, porém eles fazem parte fundamental nos processos de ideação, prototipagem e testes. Cada etapa do processo envolve um processo de auto avaliação na equipe. A flexibilização de prazos pode ser considerada em cada etapa por diversos motivos, incluindo os horários diferenciados dos alunos, a disponibilidade do grupo de pessoas e a empresa.

\section{RESULTADOS E DISCUSSÃO}

O caso de estudo apresentado neste artigo foi desenvolvido durante quatro meses, fazendo parte da disciplina de Projeto Integrador para Engenharia de Produção da UNIVESP. A equipe deste projeto foi composta por cinco alunos, que apesar de integrarem o mesmo polo presencial na cidade de Taboão da Serra, residem e trabalham em diferentes cidades da região metropolitana de São Paulo. Este fator foi determinante na preferência da comunicação de toda a equipe do projeto utilizando meios on-line e armazenamento de dados na nuvem.

\subsection{Aplicação do Design Thinking}

As etapas da metodologia de Design Thinking foram desenvolvidas conforme as atividades apresentadas na Tabela 1.

\section{Empatia}


O município de Taboão da Serra, na região da Grande São Paulo, possui cerca de 289664 habitantes (IBGE, 2019) que produzem mais de 6 mil toneladas de resíduos sólidos domiciliares mensais. Cerca de $48 \%$ destes resíduos é material orgânico e $33 \%$ apresenta a possibilidade de reciclagem (TABOÃO DA SERRA, 2009). Até 2008 a reciclagem de materiais no município era realizada por catadores, mas a Secretaria Municipal De Obras e Infraestrutura Urbana em parceria com a Fundação Nacional de Saúde e com a sociedade civil organizada elencaram como uma necessidade a criação de uma usina de reciclagem. $\mathrm{Na}$ etapa de empatia realizou-se uma pesquisa exploratória nos bairros próximos a cada integrante da equipe, buscando manter no foco central os moradores e suas necessidades. Foram relatadas diversas necessidades, as mais citadas foram relacionadas na Tabela 3.

Tabela 3 - Necessidades mais informadas pelos moradores e os possíveis projetos.

\begin{tabular}{cll}
\hline Item & Necessidades dos moradores & Possíveis projetos \\
\hline 1 & Reciclagem de resíduos sólidos & Reciclagem de resíduos sólidos \\
2 & Reforma de praças & Restauração da praça do Largo 13 de Maio \\
3 & Construção de novas praças & Praça pick-up no aeroporto de Viracopos \\
\hline
\end{tabular}

Fonte: Elaboração própria.

Após a análise a equipe optou-se pela opção 1, reciclagem de resíduos sólidos, como tema do projeto, pois teria um impacto amplo de dimensões ambientais, sociais e econômicas. Este tema possui também políticas públicas e leis que evidenciam sua necessidade para a população e o desenvolvimento sustentável. Ainda nesta etapa a equipe do projeto realizou uma pesquisa sobre o tema escolhido obtendo informações sobre os municípios das pesquisas exploratórias, suas características ambientais, sociais e econômicas. Nesta pesquisa a equipe contatou duas cooperativas de catadores de materiais recicláveis e propôs uma visita de campo que foram prontamente aceites pelas cooperativas. A Tabela 4 mostra as características da ideia e seu impacto para os moradores.

Tabela 4 - Reciclagem de resíduos sólidos

\begin{tabular}{|c|c|}
\hline \multicolumn{2}{|l|}{ Reciclagem de resíduos sólidos } \\
\hline Pontos Fortes & Pontos Fracos \\
\hline Colabora com a limpeza da cidade & $\begin{array}{l}\text { Falta informação, inclusive para os } \\
\text { trabalhadores da reciclagem }\end{array}$ \\
\hline $\begin{array}{l}\text { Sustentável, pois reutiliza materiais que seriam } \\
\text { descartados }\end{array}$ & $\begin{array}{l}\text { Apesar de existir políticas públicas existe pouca } \\
\text { ação das prefeituras }\end{array}$ \\
\hline $\begin{array}{l}\text { Criação de novos negócios e mercados para os } \\
\text { produtos reciclados }\end{array}$ & Custos de coleta, transporte e reprocessamento \\
\hline $\begin{array}{l}\text { Existem políticas públicas que apoiam projetos } \\
\text { de reciclagem }\end{array}$ & Maior custo final do produto reciclado \\
\hline Colabora na preservação do meio ambiente & $\begin{array}{l}\text { Coleta seletiva inadequada por falta de } \\
\text { informação e/ou interesse da população }\end{array}$ \\
\hline $\begin{array}{l}\text { Gera impacto social, econômico e ambiental por } \\
\text { meio do trabalho }\end{array}$ & Informalidade \\
\hline
\end{tabular}

Fonte: Elaboração própria.

\section{Definição do problema}

Desde a década de 1990 iniciou-se a formação das primeiras cooperativas de reciclagem e associações, tornando possível uma nova relação dos grupos de catadores, os municípios e poder público (DEMAJOROVIC e BESEN, 2007). A valorização e profissionalização do catador autônomo, o resgate de sua cidadania e inclusão social. A retirada dos catadores dos aterros e lixões tiveram contribuição da visão compartilhada de indivíduo, sociedade e poder público (DEMAJOROVIC e BESEN, 2007; GONÇALVES- 
DIAS e TEODÓSIO, 2006; PABLOS e BURNES, 2007). A dificuldade das cooperativas, em estabelecer vínculos e compromissos com os catadores autônomos, que preferem muitas vezes não seguir a regulamentos e conseguem alguma renda financeira, ainda que pouco, diariamente ou semanal, vendendo o material reciclável para pequenos comércios de sucatas e outros materiais (CARMO, OLIVEIRA, ARRUDA, 2006; MAZZEI e CRUBELLATE, 2007; RODRIGUEZ, 2004; SILVA, 2006; VALENTIM, 2007).

Pode-se observar um cenário adverso para as cooperativas: a alta rotatividade, falta de informação, falta de conhecimento técnico e engajamento dos cooperados revisitam toda dificuldade que as cooperativas enfrentam no seu dia-a-dia. Em 2009 a Cooperzagati, uma cooperativa de catadores foi estruturada juridicamente tornando-se responsável pela coleta seletiva do município e gerenciadora da usina de reciclagem. Atualmente, atende cerca de $13 \%$ do município de Taboão da Serra por meio da coleta seletiva municipal. Em 2018, as atividades da cooperativa evitaram que 398 toneladas de materiais recicláveis fossem para o aterro sanitário contribuindo para um meio ambiente mais equilibrado, além de gerar renda para 18 famílias. No primeiro semestre deste ano, a coleta seletiva arrecadou 259 toneladas de materiais. Com base nas informações coletadas nas entrevistas chegou-se a uma persona que está ilustrada na Figura 1, o mapa de empatia.

Figura 1 - Mapa de empatia.

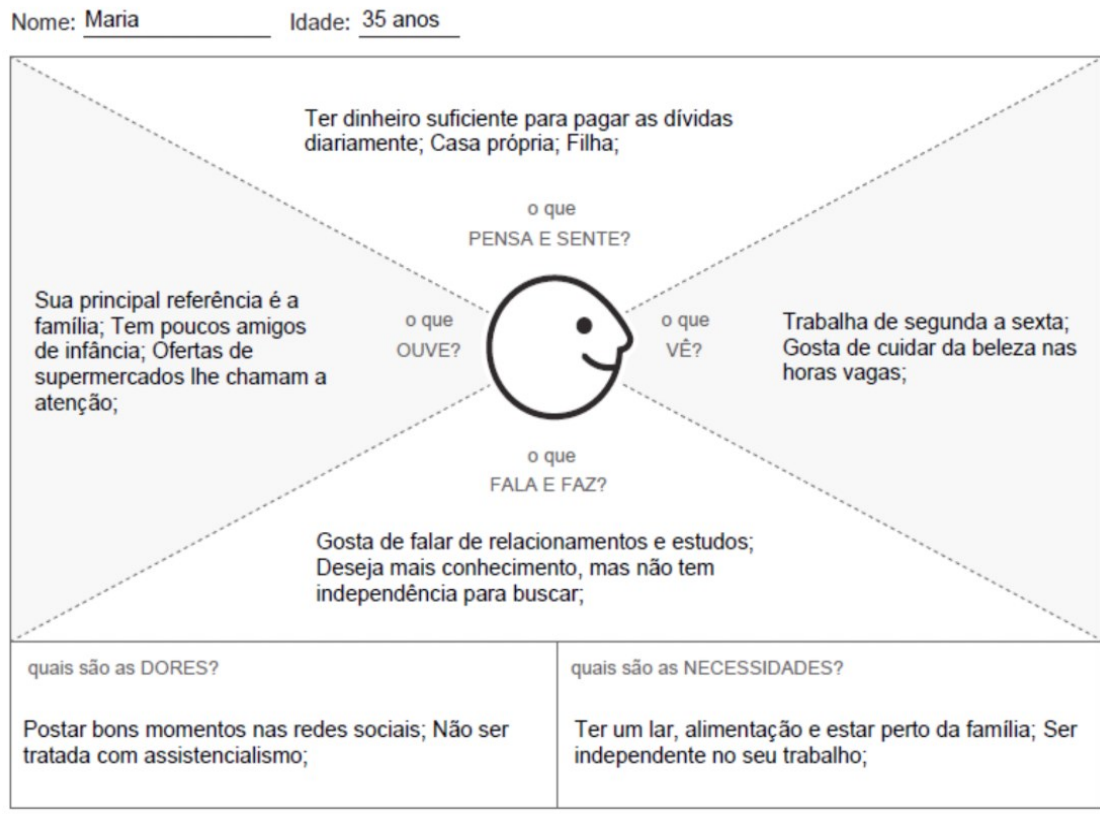

Fonte: Elaboração própria.

\section{Ideação}

As necessidades apresentadas convergiram para a implantação de um programa de integração cooperado/cooperativa segundo a norma NBR ISO 10015:2020 (ABNT, 2020). Esta ideia foi escolhida devido à baixa compreensão dos cooperados do seu papel e a informação deficiente sobre coleta seletiva e sobre a missão da cooperativa, entre outros fatores. O programa de integração busca aumentar da eficácia da coleta seletiva, promover o cooperativismo, engajar os cooperados na missão da cooperativa, oferecer informações sobre normas básicas de segurança, e integração à equipe.

\section{Prototipagem}

O protótipo deste projeto é um programa de integração, seguindo a norma NBR ISO 10015:2020 (ABNT, 2020) (ver Figura 2), baseado nas necessidades e expectativas 
ilustradas no mapa de empatia da persona. O treinamento de integração tem o objetivo de diminuir o tempo de adaptação no ambiente de trabalho, fornece confiança ao novo cooperado, proporciona segurança na execução das tarefas, auxilia no relacionamento com a equipe. Os aspectos mais relevantes a serem abordados são: histórico da organização, estrutura organizacional, expectativas, avaliações e apresentação da equipe.

Figura 2 - Ciclo de treinamento dos cooperados.

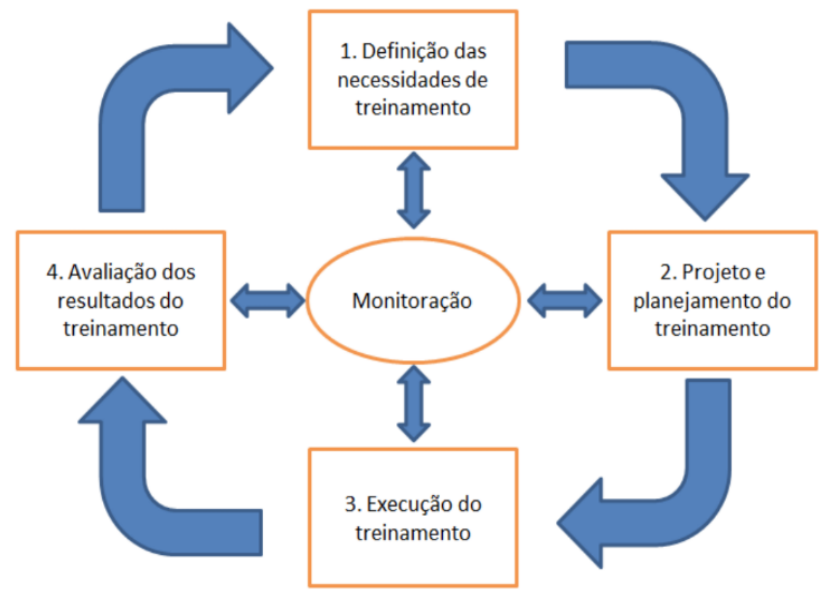

Fonte: Adaptado da NBR ISO 10015:2020 (ABNT, 2020).

Espera-se que estes treinamentos de integração possam ser executados de forma presencial, utilizando recursos audiovisuais e permitindo que aconteça na rotina semanal dos cooperados e de forma contínua.

\section{Testes}

O teste do protótipo foi realizado entre os integrantes da equipe do projeto. A partir da apresentação completa do programa de integração, selecionou-se um tópico para testar o protótipo. Durante o teste cada integrante da equipe representou um componente do programa de integração: palestrante, cooperados e observador. Avaliaram-se os diversos aspectos sobre a aplicação do programa de integração e sua melhoria contínua. Esta é uma importante ferramenta para obter devolutiva e melhorar o programa de integração. Após algumas apresentações realizadas e a análise das avaliações dos novos cooperados (na dinâmica de prototipagem) formatou-se a versão final do programa de integração.

\section{CONSIDERAÇÕES FINAIS}

O ensino de engenharia à distância enfrenta diversos desafios, especialmente na interação entre alunos e professores em tempo real. No entanto, no desenvolvimento de projetos, um planejamento adequado dos tempos de trabalho e o estabelecimento de maneiras eficientes de gerenciamento das informações obtidas em cada etapa do projeto, permite aproveitar as ferramentas tecnológicas disponíveis e potencializar esta modalidade de ensino. A integração de engenharia, design e comunidade é possível graças às devolutivas envolvidas em cada etapa da metodologia. As reuniões semanais de uma hora entre o professor-instrutor e os alunos foram essenciais para a realização das atividades propostas.

A participação de membros da comunidade em cada etapa de projetos interdisciplinares é fundamental para contextualizar as ideias propostas pelos alunos. Além disso, a devolutiva do professor-tutor e dos especialistas em cada etapa permite aprimorar 
habilidades de trabalho em equipe e toma de decisões por parte dos alunos. A metodologia de Design Thinking proposta neste trabalho permitiu formular um projeto de alto impacto na comunidade, sendo uma referência para futuros projetos desenvolvidos na modalidade à distância. Também, é essencial providenciar aos alunos métodos para a análise de políticas públicas disponíveis, pesquisa de textos acadêmicos e síntese de dados em todas as etapas do Design Thinking.

Durante a etapa de testes, a comunicação entre os membros da equipe e os usuários é vital para construir um protótipo sólido, o qual poderá ser aplicado no futuro. Diversas técnicas de testagem podem ser empregadas para determinar os pontos fortes e fracos do protótipo, na prática. O método de encenação, utilizando maquetes ou ferramentas computacionais, permite promover avaliação entre a equipe e a participação ativa dos usuários, aprimorando habilidades de toma de decisões por parte dos alunos.

\section{Agradecimentos}

Os autores agradecem o suporte da Fundação de Desenvolvimento Tecnológico da Engenharia (FDTE), Coordenação de Aperfeiçoamento de Pessoal de Nível Superior (CAPES), Conselho Nacional de Desenvolvimento Científico e Tecnológico (CNPq), Universidade de São Paulo (USP), Universidade Virtual do Estado de São Paulo (UNIVESP) e Associação Brasileira de Educação em Engenharia (ABENGE).

\section{REFERÊNCIAS}

ASSOCIAÇÃO BRASILEIRA DE NORMAS TÉCNICAS. NBR ISO 10015:2020. Gestão da qualidade - Diretrizes para gestão da competência e desenvolvimento de pessoas. Disponível em: < https://www.abntcatalogo.com.br/norma.aspx?ID=446020>. Acesso em: 15 janeiro 2020.

BROWN, Tim; WYATT, Jocelyn. Design thinking for social innovation. Development Outreach, 2010, vol. 12, no 1, p. 29-43.

CARMO, M. S; OLIVEIRA, J. A. P; ARRUDA, R. G. L. O trabalho com resíduos pelos classificadores: o papel da semântica do lixo no reconhecimento social e identidade profissional. In: EnANPAD, 30, 2006, Salvador. Anais. Salvador: ANPAD, 2006.

DEMAJOROVIC, J; BESEN, G. R. Gestão compartilhada de resíduos sólidos: avanços e desafios para a sustentabilidade. In: ENANPAD, 31, 2007, Rio de Janeiro. Anais. Rio de Janeiro: ANPAD, 2007.

GIORGETTI, M. F. Engineering and engineering technology education in Brazil. European Journal of Engineering Education, 18(4), 351-357. 1993.

GONÇALVES-DIAS, S. L. F; TEODÓSIO, A. S. S. Estrutura da cadeia reversa: "caminhos" e "descaminhos" da embalagem PET. Produção, v. 16, n. 3, p. 429-441, 2006.

INSTITUTO BRASILEIRO DE GEOGRAFIA E ESTATÍSTICA (IBGE). Panorama Taboão da Serra 2019. Disponível em: <https://cidades.ibge.gov.br/brasil/sp/taboao-daserra/panorama>. Acesso em: 17 dezembro 2019. 
INSTITUTO NACIONAL DE ESTUDOS E PESQUISAS EDUCACIONAIS (INEP). Resumo técnico do Censo da Educação Superior 2019. 2021. Disponível em: <https://download.inep.gov.br/publicacoes/institucionais/estatisticas_e_indicadores/resum o_tecnico_censo_da_educacao_superior_2019.pdf>. Acesso em: $0 \overline{7}$ agostos 2021.

LIMA, R. M., MESQUITA, D., and COELHO, L. Five years of project-based learning training experiences in higher education institutions in Brazil. 2017

MAZZEI, B. B; CRUBELLATE, J. M. Autogestão em empreendimentos econômicos solidários: um estudo comparativo de casos em cooperativas de reciclagem de Maringá-PR. In: ENANPAD, 2007, Rio de Janeiro. Anais.Rio de Janeiro: Anpad, 2007

MONTEIRO, Beany Guimarães. Design \& Inovação Social. Práticas de atuação e uso do Design em contextos locais. Araújo Filho, Targino/Thiollent, Michel Jean-Marie, Metodologia para Projetos de Extensão: Apresentação e Discussão. Universidade Federal de São Carlos (UFSCar) -São Carlos: Cubo Multimídia, 2008.

MONTEIRO, Beany; BARTHOLO, Roberto. Design and Production Engineering. Some moving frontiers of the engineering education in Brazil. En Proceedings: International Conference on Engineering Education-ICEE. 2007. p. 3-7.

PABLOS, N. P; BURNES, E. L. Bien recolectada pero mal tratada: el manejo municipal de la basura en ciudad Obregón Hermosilloy Nogales. Revista de Investigación Científica Estudios Sociales, v. 15, n. 3, p. 167-193, 2007.

RODRIGUEZ, C. À procura de alternativas econômicas em tempos de globalização: o caso das cooperativas de recicladores de lixo na Colômbia. In. SANTOS, B. S. (Org). Produzir para viver: os caminhos da produção não capitalista. 2. ed. Rio de Janeiro: Civilização Brasileira, 2004.

SILVA, P. J; BRITO, M. J. Gestão ambiental integrada: um estudo da gestão de resíduos da construção civil na cidade de Belo Horizonte-MG. In: SIMPOI, 9, 2006, São Paulo. Anais. São Paulo: SIMPOL, 2006.

STEINBECK, Reinhold. Building creative competence in globally distributed courses through design thinking. Comunicar. Media Education Research Journal, 2011, vol. 19 , no 2 .

TABOÃO DA SERRA, Secretaria Municipal de Obras e Infraestrutura (São Paulo). Taboão da Serra: Gestão de resíduos sólidos, projeto básico. Prefeitura municipal de Taboão da $\quad$ Serra, 2009 Disponível em: <http://arquivos.ambiente.sp.gov.br/cpla/2017/05/taboao-da-serra.pdf>. Acesso em: 1 setembro 2019.

VALENTIM, I. V. L. Confiar para reciclar: o significado da confiança para recicladores de resíduos sólidos de Porto Alegre. In: ENANPAD, 31, 2007, Rio de Janeiro. Anais. Rio de Janeiro: ANPAD, 2007.

VIIIARROEL G., CARLOS H., OSSAND6N N \& LASKER A. Educación en ingeniería y desarrollo, Cuardenos de Docencia no. 13 (Temuco Universidad de la Frontera). 1988 
ZINDEL, M. L., MELLO DA SILVA, J., SOUZA, J. C. F., MONTEIRO, S. B. S., and OLIVEIRA, E. C. A new approach in engineering education: the design-centric curriculum at the University of Brasília-Brazil. International Journal of Basic \& Applied Sciences, 12(5), 97-102. 2012

\title{
ENGINEERING AND PUBLIC INTEREST DESIGN: FORMULATION OF PROJECTS IN DISTANCE LEARNING ENGINEERING UNDERGRADUATE PROGRAMS USING DESIGN THINKING
}

\begin{abstract}
Interdisciplinarity is fundamental in the learning process in engineering careers. The Design Thinking methodology eases the development of projects focused on users' needs, enabling interaction between students and the community. This article proposes a method for the formulation, development, and analysis of projects using Design Thinking in distance learning education for engineering, integrating engineering, design, and community. The methodology proposed was employed for developing a training program, following the NBR ISO 10015: 2020 standards, for a recycling cooperative in Taboão da Serra. The main contribution of this work is a systematic model to develop multidisciplinary projects in engineering undergraduate courses, overcoming the limitations of distance learning and taking advantage of effective communication between students, the teachertutor, and the users.
\end{abstract}

Keywords: Design Thinking, Distance learning education, Engineering education, Entrepreneurship, Recycling. 